\title{
A First Look at Chorioamnionitis Management Practice Variation among US Obstetricians
}

\author{
Mara B. Greenberg, ${ }^{1}$ Britta L. Anderson, ${ }^{2}$ Jay Schulkin, ${ }^{2}$ \\ Mary E. Norton, ${ }^{1}$ and Natali Aziz ${ }^{1}$ \\ ${ }^{1}$ Division of Maternal Fetal Medicine, Department of Obstetrics and Gynecology, Lucile Packard Children's \\ Hospital at Stanford, Stanford University, 300 Pasteur Drive HH333, Stanford, CA 94305, USA \\ ${ }^{2}$ Department of Research, American College of Obstetricians and Gynecologists, P.O. Box 70620, Washington, WA 20024, USA
}

Correspondence should be addressed to Mara B. Greenberg, mbgreenberg@yahoo.com

Received 21 August 2012; Accepted 26 November 2012

Academic Editor: Catalin S. Buhimschi

Copyright ( 2012 Mara B. Greenberg et al. This is an open access article distributed under the Creative Commons Attribution License, which permits unrestricted use, distribution, and reproduction in any medium, provided the original work is properly cited.

\begin{abstract}
Objective. To examine practice patterns for diagnosis and treatment of chorioamnionitis among US obstetricians. Study Design. We distributed a mail-based survey to members of the American College of Obstetricians and Gynecologists, querying demographics, practice setting, and chorioamnionitis management strategies. We performed univariable and multivariable analyses. Results. Of 500 surveys distributed, $53.8 \%$ were returned, and 212 met study criteria and were analyzed. Most respondents work in group practice $(66.0 \%)$, perform $>100$ deliveries per year $(60.0 \%)$, have been in practice $>10$ years $(77.3 \%)$, and work in a nonuniversity setting $(85.1 \%)$. Temperature plus one additional criterion $(61.3 \%)$ was the most common diagnostic strategy. Over 25 different primary antibiotic regimens were reported, including use of a single agent by $30.0 \%$ of respondents. A wide range of postpartum antibiotic duration was reported from no postpartum treatment (34.5\% after vaginal delivery, $11.3 \%$ after cesarean delivery) to 48 hours of postpartum treatment (24.7\% after vaginal delivery, $32.1 \%$ after cesarean delivery). No practitioner characteristic was independently associated with diagnostic or therapeutic strategies in multivariable analysis. Conclusion. There is a wide variation in contemporary clinical practices for the management of chorioamnionitis. This may represent a dearth of level I evidence. Future prospective clinical trials may provide more evidence-based practice recommendations for diagnosis and treatment of chorioamnionitis.
\end{abstract}

\section{Introduction}

Chorioamnionitis, infection of the amniotic fluid, membranes, placenta, and/or decidua, is a common intrapartum complication, affecting up to $10 \%$ of women during labor in the USA [1-6]. Potentially serious adverse maternal sequelae of chorioamnionitis include increased risk of operative delivery, postpartum hemorrhage, and postdelivery infection $[1,6-8]$. Neonatal risks include sepsis and potential longterm consequences such as cerebral palsy [6-12]. The pathogenesis of chorioamnionitis is thought to be polymicrobial and involves bacteria that colonize the vagina including Gram-negative organisms and anaerobes. When chorioamnionitis is suspected based on clinical findings prior to delivery, administration of broad spectrum antibiotics has been shown to be effective in reducing maternal and fetal morbidity and mortality $[1-6,9]$.

Which diagnostic and therapeutic strategies are associated with optimal maternal and neonatal outcomes is unclear due to a dearth of prospective randomized trials comparing various management strategies [1-6, 13]. Clinical chorioamnionitis is traditionally diagnosed based on elevated maternal temperature associated with additional findings including maternal tachycardia, fetal tachycardia, maternal leukocytosis, uterine tenderness, and purulent amniotic fluid [1-6]. Number and type of diagnostic criteria associated with least maternal and neonatal morbidity have not been evaluated in a systematic fashion. Much of the peer-reviewed literature on chorioamnionitis either lacks a description of formal diagnostic criteria or reports utilization of elevated 
temperature plus two additional criteria $[1-5,8]$. With regard to evidence-based treatment, though randomized and observational trials have demonstrated a reduction in neonatal sepsis with intrapartum rather than postpartum treatment for chorioamnionitis, there have been few head to head comparisons of different intrapartum regimens [14-17]. In contrast, several studies have demonstrated similar efficacy among different durations of postpartum therapy, with regard to resolution of maternal infection and development of postoperative infectious complications [1821].

Likely due to limitations in the breadth of available data, guidelines are lacking regarding necessary clinical and laboratory diagnostic criteria, optimal antibiotic regimens, and duration of therapy. As a result of this uncertainty, practice patterns are likely to vary, potentially significantly. We hypothesized that obstetric care providers have a wide variety of practice patterns with regard to management of intrapartum chorioamnionitis. Identification of such variations in practice would allow us to target areas in which future investigation could be useful. In addition, for those aspects of chorioamnionitis management for which high quality data are available, it would be useful to define areas in which enhanced provider education regarding best practices might be beneficial. The purpose of this study was to determine the current practice patterns among obstetricians in the USA regarding chorioamnionitis management strategies. We also examined provider characteristics associated with the use of different diagnostic and therapeutic practices.

\section{Materials and Methods}

Questionnaires were mailed in January 2011 to 500 Fellows and Junior Fellows of the American College of Obstetricians and Gynecologists (ACOG). The potential participants were members of the Collaborative Ambulatory Research Network (CARN), a consortium established to facilitate assessment of clinical practice patterns. CARN is comprised of 1200 practicing obstetrician gynecologists who have volunteered to participate in 3-5 survey studies each year.

The pool of potential participants was quasi randomly selected from the total CARN membership. The entire CARN sample pool was divided into groups of 100 , all of which were similar with regard to mean age, gender, and ACOG district distribution. Five groups of 100 were then randomly chosen for this study sample. Each potential participant was assigned an identification number that was used to track the respondent while maintaining anonymity. A cover letter was included with the questionnaire, orienting survey recipients to the study and indicating a response deadline of approximately 3 weeks from the mailing date. Mailings also included postage paid return envelopes with the recipient identification number. All non-respondents received a second mailing of the questionnaire 5 weeks after the first mailing. A final mailing was sent approximately 5 weeks later. Questionnaires returned by July 2011 were included in the study. The survey consisted of 27 questions including 7 demographic, 9 on practice characteristics, 6 on chorioamnionitis diagnostic practices, and 5 on treatment practices. Question formats included multiple choice, yes/no, and Likert response scale. We conducted a pilot survey of 157 physicians and certified nurse midwives in June 2010 and utilized the results to eliminate ambiguity and redundancy in the questionnaire content and format. A sample of the questions on diagnostic and treatment strategies is listed in Table 1.

Student's $t$-tests were used to compare group means of continuous variables. Differences in categorical measures were assessed using Chi-squared test. Multivariable logistic regression analyses were used to control for potential confounders in assessing differences between groups. For the multivariable models, we utilized four predictor variables thought to be clinically important, regardless of significance in univariable analysis: region of practice, number of years in practice, practice volume, and practice setting. We analyzed data using R version 2.11.1 (2010-05-31, The R Foundation for Statistical Computing). We considered a two-sided alpha of $<0.05$ to be significant. Institutional review board exemption was granted at Stanford University.

\section{Results and Discussion}

3.1. Results. Of the 500 surveys distributed, 269 were returned for a $53.8 \%$ response rate. Forty were excluded due to insufficient survey completion and 17 because the respondents reported that they do not currently practice inpatient obstetrics. The remaining 212 completed surveys were included in the analysis. Participants represent 42 different US states, Canada ( 3 providers) and Bahamas (1 provider), with female and male providers equally represented and a median provider age of 51 (Table 2). The majority of providers work in private or community practice $(83.2 \%)$, have been in practice greater than 10 years $(77.3 \%)$, and perform over 100 deliveries per year $(60.0 \%)$ (Table 2).

Table 3 shows diagnostic management practices. Most providers reported using temperature plus one additional criterion for diagnosis of chorioamnionitis $(61.3 \%)$, with the most common temperature threshold being 38.0 degrees Centigrade. The majority of providers use interventions to attempt to lower maternal temperature prior to making the diagnosis of chorioamnionitis $(69.0 \%)$, including $7.1 \%$ who administer acetaminophen for this purpose prior to diagnosis. The presence of epidural analgesia influences the likelihood of diagnosing chorioamnionitis for $41.2 \%$ of providers, and $9.0 \%$ are influenced by their institution's neonatal sepsis workup policy.

Responses about treatment practices are shown in Table 3. Respondents listed over 25 different antibiotic regimens as their primary choice for treating chorioamnionitis. A regimen containing ampicillin and gentamicin is used by $65.3 \%$ of providers, while $30.0 \%$ of respondents use a singleagent regimen. A regimen without Gram-negative coverage, either ampicillin alone or cefazolin alone, is used by $14.5 \%$ of providers. A wide range of duration of postpartum antibiotic therapy was reported from no postpartum treatment (34.5\% after vaginal delivery, $11.3 \%$ after cesarean delivery) 
TABLE 1: Questions on diagnostic and treatment strategies.

Describe your most common strategy for diagnosing intrapartum chorioamnionitis.

Elevated temperature alone

$\square$ Elevated temperature plus at least one additional sign or symptom

$\square$ Elevated temperature plus at least two additional signs or symptoms

$\square$ At least one sign or symptom alone without elevated temperature

$\square$ Other:

What temperature is your threshold for diagnosing intrapartum chorioamnionitis?
$\square 37.8^{\circ} \mathrm{C}\left(100.0^{\circ} \mathrm{F}\right)$
$\square 37.9^{\circ} \mathrm{C}\left(100.2^{\circ} \mathrm{F}\right)$
$\square 38.0^{\circ} \mathrm{C}\left(100.4^{\circ} \mathrm{F}\right)$
$\square 38.1^{\circ} \mathrm{C}\left(100.6^{\circ} \mathrm{F}\right)$
$\square 38.2^{\circ} \mathrm{C}\left(100.8^{\circ} \mathrm{F}\right)$

$\square$ Other:

What strategies do you use to lower maternal temperature before deciding whether a patient meets diagnostic criteria for chorioamnionitis? Choose all that apply.

$\square$ None

$\square$ IV fluid bolus

$\square$ PO hydration

$\square$ Tylenol or other antipyretics

$\square$ External cooling (application of ice or cool cloths)

$\square$ Other:

If a patient has a fever alone, with no additional signs or symptoms of chorioamnionitis, is it likely that your decision to treat for chorioamnionitis would be influenced by whether the patient has an epidural?

$\square$ No

$\square$ Yes, I would be more likely to diagnose chorioamnionitis and treat accordingly in a patient with a fever and an epidural than one without an epidural

$\square$ Yes, I would be less likely to diagnose chorioamnionitis and treat accordingly in a patient with a fever and an epidural than one without an epidural

Do you think your institution's policy on neonatal sepsis workup influences how frequently you diagnose maternal chorioamnionitis?

$\square$ No

$\square$ Yes, I am more likely to diagnose maternal chorioamnionitis because of my institution's policy on neonatal sepsis workup

$\square$ Yes, I am less likely to diagnose maternal chorioamnionitis because of my institution's policy on neonatal sepsis workup

What is the most common antibiotic regimen you use for treating intrapartum chorioamnionitis?*
$\square$ Ampicillin
$\square$ Azithromycin
$\square$ Ancef (Cefazolin)
$\square$ Cefotetan
$\square$ Cefoxitin
$\square$ Clindamycin
$\square$ Ertapenem
$\square$ Gentamicin, daily dosing
$\square$ Gentamicin, TID dosing
$\square$ Metronidazole
$\square$ Unasyn (Ampicillin/sulbactam)
$\square$ Zosyn (Piperacillin/tazobactam) 
TABle 1: Continued.

$\square$ Ampicillin plus Gentamicin, daily dosing

$\square$ Ampicillin plus Gentamicin, TID dosing

$\square$ Ampicillin plus Gentamicin daily dosing plus Clindamycin

$\square$ Ampicillin plus Gentamicin TID dosing plus Clindamycin

$\square$ Other:

What is the most common antibiotic regimen you use for treating intrapartum chorioamnionitis in the setting of cesarean delivery?*

$\square$ Same regimen as above

$\square$ Different regimen (please check all that apply):

$\square$ Ampicillin

$\square$ Azithromycin

$\square$ Ancef (Cefazolin)

$\square$ Cefotetan

$\square$ Cefoxitin

$\square$ Clindamycin

$\square$ Ertapenem

$\square$ Gentamicin, daily dosing

$\square$ Gentamicin, TID dosing

Metronidazole

$\square$ Unasyn (Ampicillin/sulbactam)

$\square$ Zosyn (Piperacillin/tazobactam)

$\square$ Ampicillin plus Gentamicin, daily dosing

$\square$ Ampicillin plus Gentamicin, TID dosing

$\square$ Ampicillin plus Gentamicin daily dosing plus Clindamycin

$\square$ Ampicillin plus Gentamicin TID dosing plus Clindamycin

$\square$ Other:

What is your strategy for postpartum treatment after a vaginal delivery, in women diagnosed with intrapartum chorioamnionitis, in the absence of endometritis?

$\square$ No additional antibiotics after delivery

$\square$ One additional dose of antibiotics after delivery

$\square$ Continue antibiotics for 24 hours after delivery

$\square$ Continue antibiotics for 48 hours after delivery

$\square$ Continue antibiotics for 24 hours after last fever

$\square$ Continue antibiotics for 48 hours after last fever

$\square$ Other:

What is your strategy for postpartum treatment after a cesarean delivery, in women diagnosed with intrapartum chorioamnionitis, in the absence of endometritis?

$\square$ No additional antibiotics after delivery

$\square$ One additional dose of antibiotics after delivery

$\square$ Continue antibiotics for 24 hours after delivery

$\square$ Continue antibiotics for 48 hours after delivery

$\square$ Continue antibiotics for 24 hours after last fever

$\square$ Continue antibiotics for 48 hours after last fever

Other: 
TABLE 1: Continued.

Do you treat with oral antibiotics after a patient has finished her postpartum course of IV antibiotics?

$\square$ Yes

$\square$ No

* Responses were not limited to one of the choices listed but rather one or more than one antibiotic choice as needed to accurately reflect respondents' primary regimen.

to 48 hours of postpartum treatment $(24.7 \%$ after vaginal delivery, $32.1 \%$ after cesarean delivery). Sixteen percent of providers utilize oral antibiotics after completion of the intravenous regimen.

We examined the relationship between management strategies and the following provider characteristics: region of practice, length of time in practice, practice setting, and practice volume. In univariable analysis, university-based practitioners were less likely to report use of single-agent regimen $(13.8 \%$ versus $32.6 \%, P=0.04)$ and more likely to report sufficient Gram-negative coverage (100\% versus $83.4 \%, P=0.02$ ) when compared to non-university-based practitioners. Those in practice for $<10$ years were more likely to report using a regimen with sufficient Gramnegative coverage than those in practice for $>10$ years $(97.8 \%$ versus $82.0 \%, P=0.007$ ) and less likely to prescribe a course of oral antibiotics after completion of the intravenous course $(8.3 \%$ versus $24.7 \%, P=0.01)$. Region of practice was associated with likelihood of reporting use of $\leq 1$ dose of additional antibiotics after vaginal delivery compared with $>1$ dose with more providers from the Midwest treating with $\leq 1$ antibiotic dose $(67.9 \%)$ compared to providers from other regions (west $46.7 \%$, south $36.2 \%$, northeast $26.3 \%$, $P<0.001)$. No other provider characteristics were associated with differences in management strategy in univariable analysis (data not shown). In multivariable logistic regression analysis controlling for the practice volume, region, setting, and length of time in practice, no provider characteristics were independently statistically significantly associated with differences in any chorioamnionitis management strategy.

3.2. Discussion. We demonstrate a wide variation in practice patterns for management of chorioamnionitis among obstetric care providers in the USA. We identified significant heterogeneity in essentially all aspects of management, including criteria for diagnosis, influences on likelihood of making the diagnosis, choice of antibiotics, and type and duration of postpartum treatment.

This wide variety of diagnostic and treatment strategies is likely in part due to a dearth of high-quality clinical data to guide practice and indicates a need for further investigation into optimal strategies for the management of chorioamnionitis. For example, $60 \%$ of obstetricians require elevated temperature plus one additional criterion when making the diagnosis of chorioamnionitis, and a quarter of clinicians use elevated temperature alone. The latter strategy likely results in more patients being diagnosed with and treated for intrapartum infection than strategies that require additional signs or symptoms. Whether or not this lower threshold for diagnosis of chorioamnionitis is associated with fewer adverse outcomes is unclear and deserves further study.

Over two-thirds of providers utilize strategies to attempt to lower maternal temperature prior to making a diagnosis of chorioamnionitis. This may represent uncertainty by obstetricians regarding whether treatment in the setting of elevated temperature alone is associated with optimal outcomes, as well as acknowledgement that there are a variety of influences on maternal temperature in labor [1$6,22-27]$. As with other diagnostic criteria, there are no data regarding maternal or neonatal outcomes associated with initial management of maternal fever using intravenous fluids, acetaminophen, and/or external cooling prior to diagnosing chorioamnionitis and initiating antibiotics in labor.

Sixty percent of providers do not consider the presence of epidural analgesia in the diagnosis of chorioamnionitis, while the remainder are influenced by presence of epidural in making diagnostic and treatment decisions. This heterogeneity of management styles reflects the lack of consensus on best practices regarding the complex relationship between epidural, maternal fever, and neonatal outcomes, despite multiple publications addressing this issue [22-27]. While current literature suggests that elevated maternal temperature associated with epidural use is not associated with neonatal sepsis, a consensus regarding how presence of epidural should inform chorioamnionitis diagnostic threshold has yet to be reached [22-27].

The vast majority of obstetricians surveyed do not take their institution's neonatal sepsis workup policy into account when diagnosing chorioamnionitis. However, of the $7 \%$ of providers who are influenced by such policies, more than two-thirds report that they are less likely to diagnose and treat clinically suspected chorioamnionitis as a result of their institution's policy. Presumably this indicates a degree of uncertainty regarding the cost-benefit ratio of exposing neonates to invasive procedures, given the lack of an evident number needed to treat to prevent neonatal harm when the clinical diagnosis of chorioamnionitis is uncertain.

A 2002 Cochrane Database Review indicated that there are insufficient data on which to base a recommendation for optimal antibiotic regimens in the setting of chorioamnionitis [13]. Not surprisingly, our results demonstrate a wide variety of antibiotic regimens in common use. Most of these regimens contain sufficient Gram-negative coverage and consist of broad spectrum antibiotics that cover for betalactamase producing aerobes and anaerobes, as has been recommended by expert consensus and nonrandomized published studies $[1-6,9,13-16,22-27]$. However, 15\% 
TABLE 2: Respondent characteristics.

\begin{tabular}{|c|c|}
\hline Respondent characteristics & $N=212$ \\
\hline Female & $103(48.8 \%)$ \\
\hline Male & $109(51.2 \%)$ \\
\hline Median age (IQR) & $51(43-60)$ \\
\hline \multicolumn{2}{|l|}{ Physician ethnicity } \\
\hline Non-Hispanic White & $173(82.0 \%)$ \\
\hline Other & $38(18.0 \%)$ \\
\hline \multicolumn{2}{|l|}{ Number of deliveries annually } \\
\hline$<100$ & $84(40.0 \%)$ \\
\hline$>100$ & $126(60.0 \%)$ \\
\hline \multicolumn{2}{|l|}{ Region of US ${ }^{a}$} \\
\hline West & $46(22.0 \%)$ \\
\hline Midwest & $54(25.8 \%)$ \\
\hline South & $69(33.0 \%)$ \\
\hline Northeast & $38(18.2 \%)$ \\
\hline \multicolumn{2}{|l|}{ Practice location } \\
\hline Suburban & $106(50.2 \%)$ \\
\hline Urban & $75(35.5 \%)$ \\
\hline Rural & $25(11.8 \%)$ \\
\hline Other & $5(2.5 \%)$ \\
\hline \multicolumn{2}{|l|}{ Predominant patient insurance type } \\
\hline Private & $154(73.3 \%)$ \\
\hline Public & $55(26.2 \%)$ \\
\hline Uninsured & $1(0.5 \%)$ \\
\hline \multicolumn{2}{|l|}{ Predominant patient ethnicity } \\
\hline Non-Hispanic White & $148(70.8 \%)$ \\
\hline Hispanic White & $32(15.3 \%)$ \\
\hline Other & $29(13.9 \%)$ \\
\hline \multicolumn{2}{|l|}{ Years in practice } \\
\hline $0-5$ & $5(2.4 \%)$ \\
\hline $6-10$ & $43(20.3 \%)$ \\
\hline $11-15$ & $43(20.3 \%)$ \\
\hline $16-20$ & $25(11.8 \%)$ \\
\hline $21-25$ & $34(16.0 \%)$ \\
\hline $26-30$ & $27(12.7 \%)$ \\
\hline$>30$ & $35(16.5 \%)$ \\
\hline \multicolumn{2}{|l|}{ Practice setting } \\
\hline Private or community & $174(83.2 \%)$ \\
\hline University or academic & $29(13.9 \%)$ \\
\hline Government & $5(2.4 \%)$ \\
\hline Other & $1(0.5 \%)$ \\
\hline \multicolumn{2}{|l|}{ Practice type } \\
\hline Obgyn partnership/group & $140(66.0 \%)$ \\
\hline University/teaching institution & $26(12.3 \%)$ \\
\hline Solo practice & $38(17.9 \%)$ \\
\hline Laborist/hospitalist & 0 \\
\hline Other & $8(3.8 \%)$ \\
\hline
\end{tabular}

${ }^{a}$ Location of practice divided into four regions for purposes of analysis, according to the Centers for Disease Control and Prevention "Geographic Regions of the United States," http://www.cdc.gov/.

of providers use ampicillin or cefazolin alone, regimens that do not contain Gram-negative coverage and are theoretically insufficient for covering the spectrum of microbes commonly seen with chorioamnionitis. We found that use of a regimen with insufficient Gram-negative coverage is associated with practice type and duration, with community physicians and those in practice for $>10$ years more likely to use such a regimen than those in university practice and more recently in training.

While use of a single agent appears to be common practice ( $30 \%$ of respondents), no studies have compared the use of a single agent to a multidrug regimen for treatment of chorioamnionitis. Use of a single agent is also associated with practice type, with fewer physicians in university practice reporting use of a single agent as compared to those in community practice. These discrepancies may be indicative of variation in management styles between university and community practices.

Several studies have investigated the association between duration of postpartum therapy, resolution of maternal infection, and development of postoperative infectious complications [18-21]. While randomized trials have not demonstrated a difference in efficacy between "traditional" 24-48 hour postpartum courses and "abbreviated" courses consisting of $\leq 1$ postpartum dose of antibiotics, the majority of providers continue to use $24-48$ hour courses. Unlike other practice patterns studied, region of practice but not practice type is associated with likelihood of adopting an abbreviated postpartum antibiotic course. While most obstetricians do not use oral antibiotic therapy after completion of an intravenous postpartum course, a significant minority $(16 \%)$ uses oral antibiotics to complete a longer treatment course. This practice is in contrast with randomized data demonstrating lack of benefit of such a strategy [28].

While this study describes clear differences in the management of chorioamnionitis amongst a diverse group of obstetric providers, it is not without limitations. These include a relatively small sample size, which may decrease our ability to detect potential differences in management practices attributable to variability in provider characteristics. Similarly, our findings may not be generalizable to practitioners outside of the ACOG membership and the demographic included. The response rate of 54\% may have led to nonresponse bias, though this is near the upper end of the $35 \%-60 \%$ response rate typical of previous ACOG surveys $[29,30]$. Additionally among those surveyed, a wide variety of practice settings and geographic regions were represented. Due to the constraints of a postal-based survey strategy, we were also limited in our ability to capture more comprehensive details about chorioamnionitis management strategies and their associated outcomes or about the risk profiles of respondent's patient populations. Many providers likely have a more nuanced approach to the diagnosis and treatment of chorioamnionitis than can be appreciated based on the brief closed-ended survey approach employed.

\section{Conclusions}

This study is the first to report on chorioamnionitis management patterns among US obstetricians. The heterogeneity of practice patterns we demonstrate has several potential implications. Many aspects of variation in management 
TABle 3: Diagnostic and treatment strategies.

Diagnostic strategies

$N=212$

Diagnosis based on

Temperature alone

$56(26.4 \%)$

Temperature plus one additional criterion

$130(61.3 \%)$

Temperature plus two additional criteria

$16(7.6 \%)$

Other

$10(4.7 \%)$

Most common temperature threshold (degrees Centigrade)

37.9

$6(2.8 \%)$

38.0

$154(73.0 \%)$

38.1

$23(10.9 \%)$

38.2

$18(8.5 \%)$

Other

$10(4.7 \%)$

Strategies used to lower temperature prior to diagnosis

None

$65(31.0 \%)$

Intravenous fluids

$124(59.0 \%)$

Acetaminophen

$15(7.1 \%)$

Other

$6(2.9 \%)$

Influenced by presence of epidural in making diagnosis

No

$124(58.8 \%)$

More likely to diagnose

$10(4.7 \%)$

Less likely to diagnose

$77(36.5 \%)$

Neonatal sepsis workup required for all chorioamnionitis diagnoses

Yes

$170(83.3 \%)$

No

$34(16.7 \%)$

Influenced by neonatal sepsis workup policy in making diagnosis

No

$191(91.0 \%)$

More likely to diagnose

$5(2.3 \%)$

Less likely to diagnose

$14(6.7 \%)$

Treatment Strategies

$N=212$

Primary treatment regimen

Ampicillin and gentamicin \pm additional agent

$135(65.2 \%)$

Single agent

$62(30.0 \%)$

Includes Gram-negative coverage

$177(85.5 \%)$

Does not include Gram-negative coverage

$30(14.5 \%)$

Change regimens for cesarean delivery

Yes

$99(46.9 \%)$

No

$112(53.1 \%)$

Postpartum treatment strategy after vaginal delivery

No additional antibiotics

$73(34.6 \%)$

1 additional dose

$20(9.5 \%)$

24 hours postpartum

$56(26.5 \%)$

24 hours afebrile

48 hours postpartum

$52(24.7 \%)$

48 hours afebrile

$4(1.9 \%)$

Other

$3(1.4 \%)$

Postpartum treatment strategy after cesarean delivery

No additional antibiotics

$24(11.3 \%)$

1 additional dose 
Table 3: Continued.

$\begin{array}{lc}24 \text { hours afebrile } & 17(8.0 \%) \\ 48 \text { hours postpartum } & 68(32.1 \%) \\ 48 \text { hours afebrile } & 16(7.6 \%) \\ \text { Other } & 2(0.9 \%) \\ \text { Treat with oral antibiotics after intravenous course completed } & 34(16.2 \%) \\ \text { Yes } & 176(83.8 \%) \\ \text { No } & \end{array}$

strategy may reasonably be thought to represent lack of highquality data necessary to guide practice in a coherent fashion. These understudied aspects of care include number and type of diagnostic criteria, influence of epidural on diagnosis, and type of antibiotic regimen. While some heterogeneity in practice may be acceptable, such as in the use of numerous antibiotics that cover the same bacteria but have varying prices, availability, ease of use, and tolerability, studies determining optimal maternal and neonatal outcomes associated with particular management strategies in these understudied areas may be helpful to inform best practices. Data regarding which strategies are associated with decreased rates of neonatal sepsis and improvements in long-term child health are important to guide providers in these areas as these are associated with the most current uncertainty. With regard to several management issues that have already been studied in randomized trials, such as duration and type of postpartum therapy, our results indicate that efforts should be made to increase dissemination of best practice guidelines across obstetric demographics.

\section{Acknowledgments}

This study was funded in part by Grant no UA6MC19010, through the Maternal and Child Health Research Programs, Health Resources and Services Adminstration, U.S. Department of Health and Human Services, presented at the Society for Maternal Fetal Medicine 32nd Annual Meeting, February 6-11, 2012, Dallas, TX.

\section{References}

[1] A. T. N. Tita and W. W. Andrews, "Diagnosis and management of clinical chorioamnionitis," Clinics in Perinatology, vol. 37, no. 2, pp. 339-354, 2010.

[2] L. C. Gilstrap III and S. M. Cox, "Acute chorioamnionitis," Obstetrics and Gynecology Clinics of North America, vol. 16, no. 2, pp. 373-379, 1989.

[3] D. E. Soper, C. G. Mayhall, and H. P. Dalton, "Risk factors for intraamniotic infection: a prospective epidemiologic study," American Journal of Obstetrics and Gynecology, vol. 161, no. 3, pp. 562-566, 1989.

[4] E. R. Newton, "Chorioamnionitis and intraamniotic infection," Clinical Obstetrics and Gynecology, vol. 36, no. 4, pp. 795-808, 1993.

[5] J. W. Riggs and J. D. Blanco, "Pathophysiology, diagnosis, and management of intraamniotic infection," Seminars in Perinatology, vol. 22, no. 4, pp. 251-259, 1998.
[6] R. K. Edwards, "Chorioamnionitis and labor," Obstetrics and Gynecology Clinics of North America, vol. 32, no. 2, pp. 287296, 2005.

[7] J. C. Hauth, L. C. Gilstrap, G. D. V. Hankins, and K. D. Connor, "Term maternal and neonatal complications of acute chorioamnionitis," Obstetrics and Gynecology, vol. 66, no. 1, pp. 59-62, 1985.

[8] D. J. Rouse, M. Landon, K. J. Leveno et al., "The maternalfetal medicine units cesarean registry: chorioamnionitis at term and its duration-relationship to outcomes," American Journal of Obstetrics and Gynecology, vol. 191, no. 1, pp. 211216, 2004.

[9] R. S. Gibbs and P. Duff, "Progress in pathogenesis and management of clinical intraamniotic infection," American Journal of Obstetrics and Gynecology, vol. 164, no. 5 I, pp. 1317-1326, 1991.

[10] J. M. Alexander, D. M. McIntire, and K. J. Leveno, "Chorioamnionitis and the prognosis for term infants," Obstetrics and Gynecology, vol. 94, no. 2, pp. 274-278, 1999.

[11] J. G. Shatrov, S. C. M. Birch, L. T. Lam, J. A. Quinlivan, S. McIntyre, and G. L. Mendz, "Chorioamnionitis and cerebral palsy: a meta-analysis," Obstetrics and Gynecology, vol. 116, no. 2, pp. 387-392, 2010.

[12] N. Aziz, Y. W. Cheng, and A. B. Caughey, "Neonatal outcomes in the setting of preterm premature rupture of membranes complicated by chorioamnionitis," Journal of Maternal-Fetal and Neonatal Medicine, vol. 22, no. 9, pp. 780-784, 2009.

[13] L. Hopkins and F. Smaill, "Antibiotic regimens for management of intraamniotic infection," Cochrane Database of Systematic Reviews, no. 3, Article ID CD003254, 2002.

[14] R. S. Sperling, R. S. Ramamurthy, and R. S. Gibbs, "A comparison of intrapartum versus immediate postpartum treatment of intra-amniotic infection," Obstetrics and Gynecology, vol. 70, no. 6, pp. 861-865, 1987.

[15] R. S. Gibbs, M. J. Dinsmoor, E. R. Newton, and R. S. Ramamurthy, "A randomized trial of intrapartum versus immediate postpartum treatment of women with intraamniotic infection," Obstetrics and Gynecology, vol. 72, no. 6, pp. 823-828, 1988.

[16] L. C. Gilstrap, K. J. Leveno, S. M. Cox, J. S. Burris, M. Mashburn, and C. R. Rosenfeld, "Intrapartum treatment of acute chorioamnionitis: impact on neonatal sepsis," American Journal of Obstetrics and Gynecology, vol. 159, no. 3, pp. 579$583,1988$.

[17] M. C. Maberry and L. C. Gilstrap, "Intrapartum antibiotic therapy for suspected intraamniotic infection: impact on the fetus and neonate," Clinical Obstetrics and Gynecology, vol. 34, no. 2, pp. 345-351, 1991.

[18] S. J. Chapman and J. Owen, "Randomized trial of single-dose versus multiple-dose cefotetan for the postpartum treatment of intrapartum chorioamnionitis," American Journal of Obstetrics and Gynecology, vol. 177, no. 4, pp. 831-834, 1997. 
[19] R. K. Edwards and P. Duff, "Single additional dose postpartum therapy for women with chorioamnionitis," Obstetrics and Gynecology, vol. 102, no. 5, pp. 957-961, 2003.

[20] M. A. Turnquest, H. Y. How, C. R. Cook et al., "Chorioamnionitis: is continuation of antibiotic therapy necessary after cesarean section?" American Journal of Obstetrics and Gynecology, vol. 179, no. 5, pp. 1261-1266, 1998.

[21] C. Berry, K. A. Hansen, and J. F. McCaul, "Abbreviated antibiotic therapy for clinical chorioamnionitis: a randomized trial," Journal of Maternal-Fetal Medicine, vol. 3, no. 5, pp. 216-218, 1994.

[22] B. L. Leighton and S. H. Halpern, "The effects of epidural analgesia on labor, maternal, and neonatal outcomes: a systematic review," American Journal of Obstetrics and Gynecology, vol. 186, no. 5, pp. S69-S77, 2002.

[23] L. Goetzl, A. Cohen, F. Frigoletto Jr., J. M. Lang, and E. Lieberman, "Maternal epidural analgesia and rates of maternal antibiotic treatment in a low-risk nulliparous population," Journal of Perinatology, vol. 23, no. 6, pp. 457-461, 2003.

[24] O. Apantaku and V. Mulik, "Maternal intra-partum fever," Journal of Obstetrics and Gynaecology, vol. 27, no. 1, pp. 1215, 2007.

[25] L. E. Riley, A. C. Celi, A. B. Onderdonk et al. et al., "Association of epidural-related fever and noninfectious inflammation in term labor," Obstetrics and Gynecology, vol. 117, no. 3, pp. 588595, 2011.

[26] E. Lieberman, J. M. Lang, F. Frigoletto Jr., D. K. Richardson, S. A. Ringer, and A. Cohen, "Epidural analgesia, intrapartum fever, and neonatal sepsis evaluation," Pediatrics, vol. 99, no. 3, pp. 415-419, 1997.

[27] F. A. de Orange, R. Passini Jr., M. M. Amorim, T. Almeida, and A. Barros, "Combined spinal and epidural anaesthesia and maternal intrapartum temperature during vaginal delivery: a randomized clinical trial," British Journal of Anaesthesia, vol. 107, no. 5, pp. 762-768, 2011.

[28] M. J. Dinsmoor, E. R. Newton, and R. S. Gibbs, "A randomized, double-blind, placebo-controlled trial of oral antibiotic therapy following intravenous antibiotic therapy for postpartum endometritis," Obstetrics and Gynecology, vol. 77, no. 1, pp. 60-62, 1991.

[29] D. M. Kissin, M. L. Power, E. B. Kahn et al., "Attitudes and practices of obstetrician-gynecologists regarding influenza vaccination in pregnancy," Obstetrics and Gynecology, vol. 118, no. 5, pp. 1074-1080, 2011.

[30] K. A. Matteson, B. L. Anderson, S. B. Pinto, V. Lopes, J. Schulkin, and M. A. Clark, "Practice patterns and attitudes about treating abnormal uterine bleeding: a national survey of obstetricians and gynecologists," American Journal of Obstetrics and Gynecology, vol. 205, no. 4, pp. 321.e1-321.e8, 2011. 


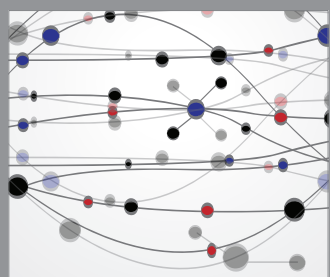

The Scientific World Journal
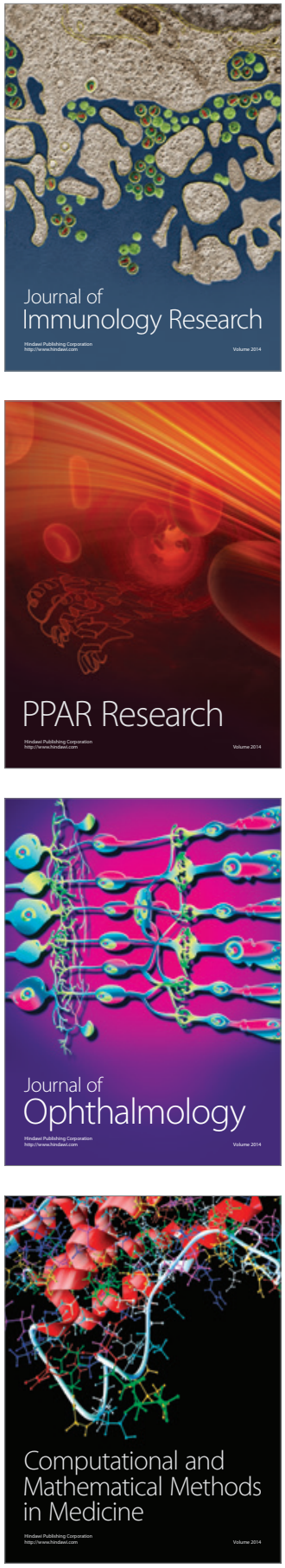

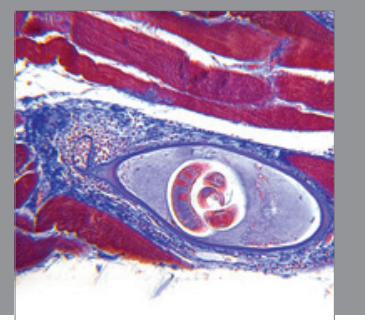

Gastroenterology

Research and Practice
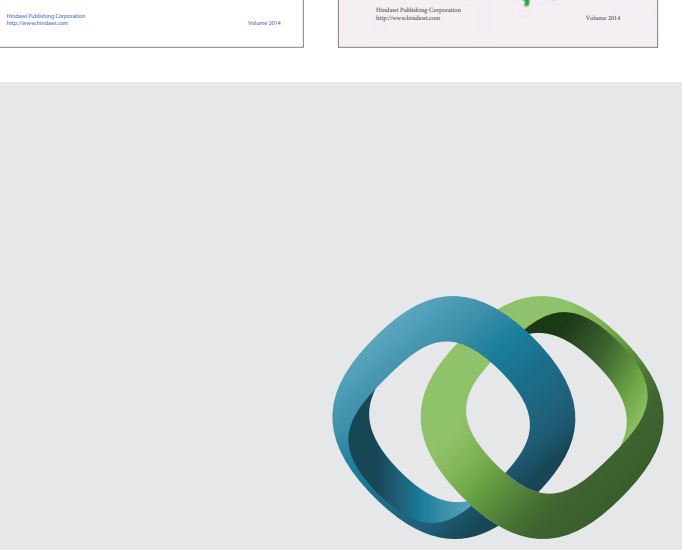

\section{Hindawi}

Submit your manuscripts at

http://www.hindawi.com
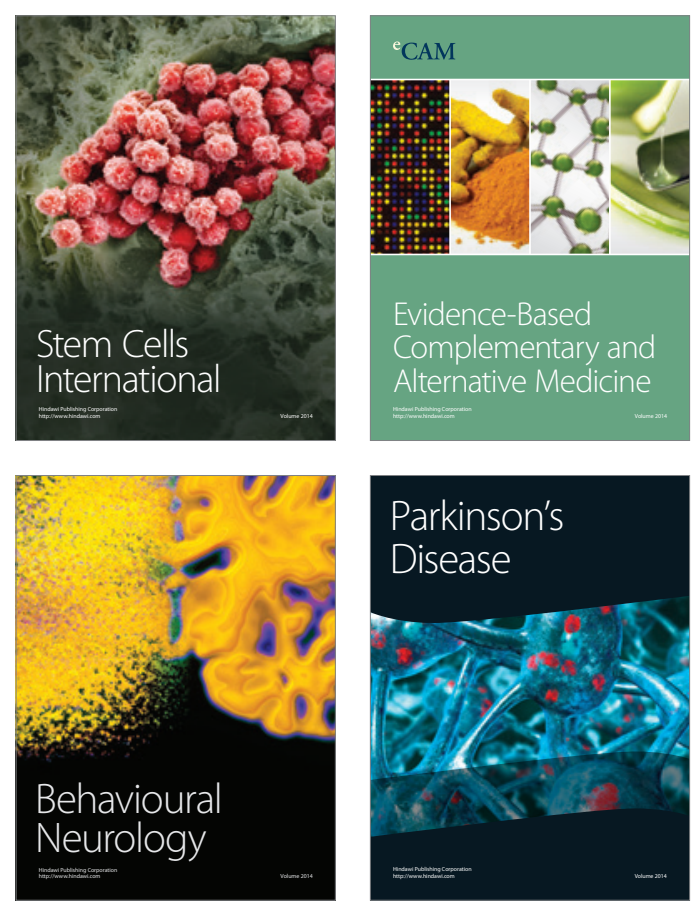

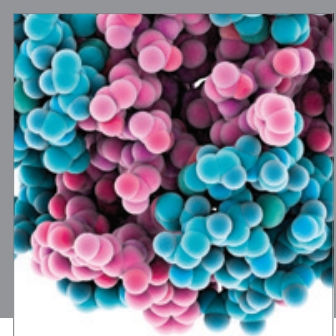

Journal of
Diabetes Research

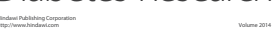

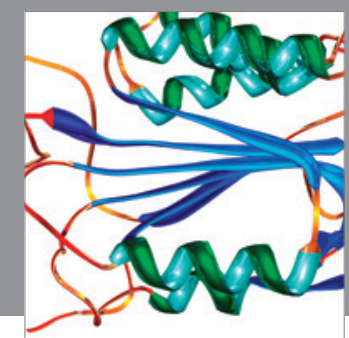

Disease Markers
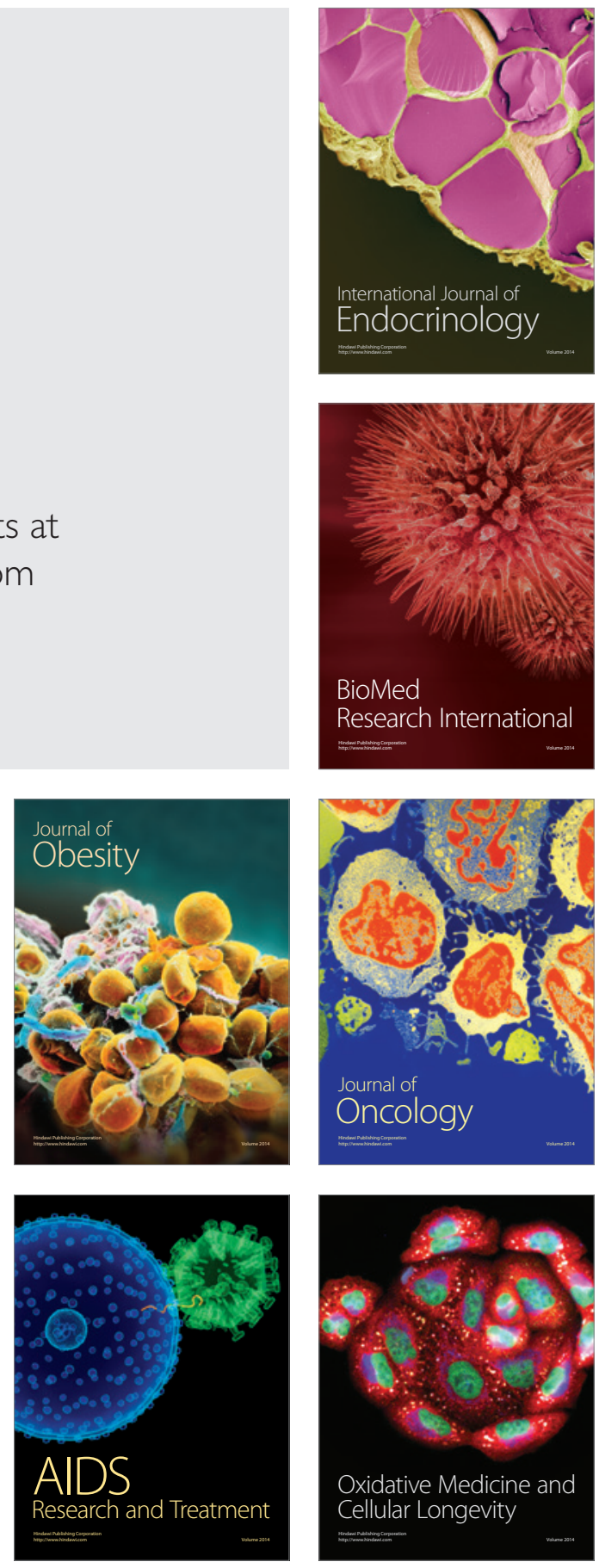